\title{
Investigation of principles of simulation of space-time processing of wideband signals
}

\author{
Vasiliy N. Vasyukov ${ }^{1}$, Daria N. Zima ${ }^{1}$, Igor F. Lozovskiy ${ }^{2}$, Yury V. Morozov ${ }^{1}$, \\ Aleksey A. Murasev ${ }^{1}$, Ivan A. Pichkov ${ }^{1}$, Mikhail A. Rajfeld ${ }^{1}$, Daria O. Sokolova ${ }^{1}$ and \\ Aleksandr A. Spektor ${ }^{1}$ \\ ${ }^{1}$ Novosibirsk State Technical University, Novosibirsk, Russia \\ ${ }^{2}$ JSC "Scientific Research Institute of Measuring Instruments - Novosibirsky Plant Named After Komintern", Novosibirsk, \\ Russia
}

\begin{abstract}
The paper states the principles of simulation of wideband signals applied in a surveillance radar. The resource of the radar model is analyzed with respect to sample rate of processed signals and required memory size as generated signals, noises, active and passive interferences demand a lot of memory. It is proposed to simulate only active intervals of operation of a pulse radar at zero frequency in the frequency domain.
\end{abstract}

Keywords

Radiolocation, simulation, interference, sample rate, wideband signal, space-time processing.

\section{Introduction}

A mathematical model of a surveillance radar system (SRS) with digital phased-array antenna (DPAA) where wideband signals are applied should be built with respect to features of solved problems and capabilities of modern computers. There are a lot of research works on radar simulation in Russia and abroad. General principles of radar simulation based on the radar equation are stated in $[1,2]$.

A generalized structure of a program for radar simulation is stated in [3]. The authors of [3] say that their model exactly reflect physical processes in a medium where targets fly with respect to all possible types of noises and interferences and correctly describe algorithms of processing signals reflected from targets. The model operates with sampled signals, noises and interferences that are not averaged as in previous models. The simulation program divided into modules gives possibility to analyze different variants of algorithms of radar signal processing in presence of various noises and interferences. Besides, it is possible to process radar signal both at carrier frequency and at zero frequency in real time or in the post-processing mode depending on computer characteristics.

The model is aided on investigation of future radars. The high experience of SRS simulation is mentioned. It is noted that earlier models were oriented on definite types of SRS not on future types.

SDM-2021: All-Russian conference, August 24-27, 2021, Novosibirsk, Russia

@yu.morozov@corp.nstu.ru (Y.V. Morozov)

(c) (1) $\odot 2021$ Copyright for this paper by its authors. Use permitted under Creative Commons License Attribution 4.0 International (CC BY 4.0).

CEUR Workshop Proceedings (CEUR-WS.org) 
The capabilities of SRS simulation based on numerical models are analyzed in [4]. This paper dedicated to SRS of terahertz range contains useful general simulation principles. The numerical model proposed in [4] includes parameters input, antenna simulation, scatters characteristics calculation, effective scattering surface (ESS) calculation, signal analysis and results output.

The input parameters are divided into the groups: radar parameters, target parameters, atmosphere parameters, The radar parameters include wavelength, pulse width, pulse frequency, antenna diagram, effective antenna surface, pulse power. The target parameters include only distance, velocity vector and azimuth. Hence, this program can operate only with surface targets. The final result of simulation is the signal-to-noise ratio (SNR) providing target detection with a given probability for a fixed false-alarm probability. The disadvantage of this model is the necessity to use enormous Maxwell differential equations.

The mathematical model of a radar system directed on target classification is considered in [5]. The model is able to calculate the maximal distance and SNR by means of the radar equation. The Bayes approach is used for the target classification.

To reduce the required computer memory volume and calculation time it is proposed to simulate a target and a medium with FPGA [6]. The advantages and disadvantages of target simulators produced by the key manufacturers of radio instrumentation including Keysight, Anritsu, National Instruments are analyzed. The main disadvantage of these simulators is a very high price. Cheaper simulators functional capabilities are limited by a small number of simulated targets or disability to control of some parameters.

The simulation program described in [7] is similar to the model in [3] but it can operate only with narrowband signals with $10 \mathrm{MHz}$ bandwidth.

The baseband radar model for radiolocation and telecommunications is proposed in [8]. The simulation is implemented in time domain but it is not suitable for radars with wideband signals.

The baseband radar simulation is also considered in [9] for narrowband signals. The main attention is focused on the mathematical description of interferences, signals transformation from high-frequency to baseband quadrature components.

Successes and problems of a wideband radar system are analyzed in [10]. The general formulas of a baseband signal reflected from a target are considered. The advantages of wideband radar stations are shown for target detection, coordinates measurement and classification.

The theoretical bases of radar signals spectrums transformation in a medium with a target are considered [11]. The simulation is limited on by DPAA size $16 \times 16$ elements.

The linear frequency modulation (LFM) signal with Additive White Gauss Noise (AWGN) reflected from a multi-scatter target is discussed in [12]. There is a high demand on memory size.

The radar simulation system proposed in [13] consists of three main components including a computer with a user interface generating a simulation scenario, modules with built-in processors for calculation of signals in a medium and FPGA for baseband signals generation.

The radar model for a student tutorial on radar bases is presented in [14]. The model has limited capabilities. The signal bandwidth is no more than $2 \mathrm{MHz}$. Hence the model cannot operate with wideband signals with the bandwidth $100 \mathrm{MHz}$ and more. The model can operate only with single-point targets. There are a few interferences like mountains and no active interferences. The radar system model presented in [15] was developed in Simulink by a student from the engineering faculty of the Moratuva University, Sri-Lanka. The model generates only 
a radio impulse with pulse amplitude modulation (PAM). It simulates AWGN and delay from target. The simplest radar system model is stated in [16]. It is a calculator of delay time from distance in miles. It is evident that the models $[14,15,16]$ are unsuitable for the scientific research.

All the considered models of radar stations with DPAA solve main tasks that appear when methods and aids of radar signal processing are developed. A few models are directed on simulation of wideband signals scattered by a multi-scatter target. Usually very expensive models requiring multi-processor systems are proposed. Therefore, a complete radar station model which is able to operate with wideband signals and interferences and provide demanded features is out of free open access. The model proposed in the present paper is directed on development and investigation of a radar station based on operating with wideband signals and interferences.

\section{Principles of simulation of a radar station with DPAA}

Radar is featured by using high-frequency signals. Such signals can be sampled with very high sample rate demanding high speed and big Random-Access Memory (RAM) volume of a computer. To provide operation of a SRS mathematical model with DPAA at a common computer with medium speed and not big volume of RAM, it is necessary to execute simulation at baseband (zero frequency).

A pulse radar station is featured by long time intervals between pulses. The simulation of processes in pauses between pulses can lead to long calculation time with almost no useful information. It is advisable to simulate only those intervals of signals and interferences that correspond to a pulse position. To except edge effects, the duration of a simulated interval should be increased by additional intervals before a pulse and after it.

When an interval with no signal is deleted from a radar model it is impossible to control falsealarm probability. If false-alarm probability is about $10^{-7}$, it is difficult to estimate experimentally a detection threshold. Hence, it is possible to control of the detection threshold with respect to the theoretical calculation results $[17,18]$.

A radar mathematical model should take into account any number of active interference sources placed at different space points. Interferences are simulated as noises with a spectrum wider than a signal spectrum. It is proposed to simulate passive interferences as reflectors clouds near a target or reflectors distributed at the ground surface.

A mathematical model of a passive interference can be described by a Gauss process with a bandwidth depending on a useful signal spectrum. The model should simulate correlations between signal periods for providing the capability of their attenuation and adaptation to wind speed. Passive interference is suppressed by the first-order and second-order over-period subtraction.

The mathematical model should help to analyze the radar operation when a target is detected from a pulse train.

The proposed model simulated an SRS with a wideband pulse signal. If the bandwidth is $100 \mathrm{MHz}$, than the distance resolution is $1.5 \mathrm{~m}$. Then a target size is more than the resolution, and a target surface has many scattering points. Each scattering point has its cross-section, 
phase drops depending on a scattering point type. Target orientation can change its scattering features.

The detector in the proposed model has an operating window for statistical data accumulation. The window size is matched with a target size. If a target type is changed the window size is adapted. If resolution is tested three windows are formed. Two of them correspond to known targets coordinates while the third one is placed to the midpoint. If the targets are resolved they are not detected in the middle window else they are detected in all three windows.

It is important to introduce geometry into the model for taking into account the features of the radar and its antenna and a target with controllable coordinates and orientation. An active interference source should have the controllable parameters including their coordinates and power spectral density (PSD).

The main attention is focused on signal transformation when it is transmitted from an antenna to a target, then received by the antenna with following digital processing. The sounding signal is generated with respect to an antenna diagram.

The features caused by spectrum expansion up to $100 \mathrm{MHz}$ almost do not influence on the electromagnetic field forming in the far-field region. Thus, common methods are used for calculating power flux of an incident way with respect to the transmitted power and distance between the radar and the target and the angle between the antenna diagram and the normal to the array antenna surface.

A target forming a reflected signal is described as a linear stationary filter with the gain depending on a target type, the number and positions of scattering points and its orientation relative to a radar station.

The received signal is found at one of DPAA elements. Then it is transferred to other DPAA elements with respect to delays. This operation is implemented in the frequency domain to avoid complicated interpolation calculations in the time domain. The transfer of the received signal to all antenna elements leads to appearance of the signal as a function of time and space coordinates defined by positions of antenna elements.

The received signal is processed by an adaptive space-time filter to reduce active interferences.

If an array is flat, a space-time signal is a function of three variables including time and to space coordinates. The received signal at all elements is described by the 3D rectangular area sampled with certain signal values in nodes. The 3D FFT gives a 3D spectrum depending on two space frequencies and time frequency. The SNR is maximized by using a matched filter with the gain found from 3D spectrum of a signal and interferences. The interference spectrum is used for adaptation when radar transmitter is switched off for accumulating a training signal

The signal at the space-time filter output is formed in the frequency domain. Then this signal is transformed by the Inverse Fast Fourier Transform (IFFT) to the space-time signal with attenuated active interferences and thermal noise. Then a 1D time-domain signal is extracted from the 3D space-time signal at fixed values of space coordinates that correspond to the maximal SNR. The 1D time-domain signal is used by a detector and an instrument for measuring the arrival time of the reflected signal.

The arrival time estimation for measuring distance until a target is specific because of the wideband signal high resolution. If a target has main scattering points then it is impossible to measure a received signal with respect to the peak position. The truth target position is the average point between the nearest to the radar and the furthest from the radar scatters. The 
measured and truth values give possibility to find measurement errors.

When a passive interference occurs, the signal after suppression of an active interference is processed to suppress the passive interference by an adaptive over-period subtractor of the first or second order.

The proposed model of the radar system with DPAA is based on the following principles: all signals are simulated by complex envelopes; only active intervals of sounding signal pulses are simulated and long pauses between sounding pulses are not simulated; all operations on signals are performed in the frequency domain; space processing cannot be separated from time processing.

\section{User interface of the proposed model}

The proposed model is implemented as a program with the control panel for setting the initial configuration parameters including model structure, sounding signal parameters, radar characteristics, target characteristics, noise and interference parameters. Targets and active interferences sources are described by electronic tables with their initial coordinates, velocity projections and etc.

The control panel consists of the list of controls groups, lists for selection of files with descriptions of targets and active interferences, buttons for control of model run and stop and loading initial configuration parameters (Figure 1).

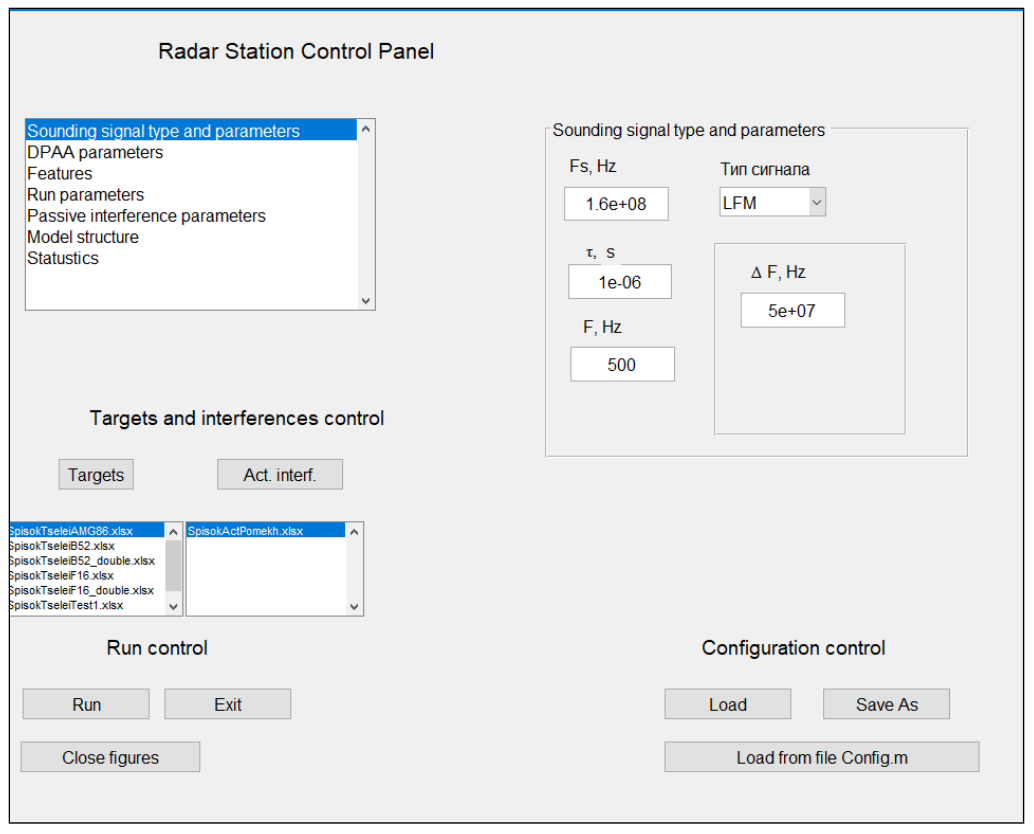

Figure 1: Control panel for radar station simulation. 


\section{Coordinates transformation for the radar station with DPAA model}

When an SRS is simulated it is necessary to take into account geometrical aspects because of many flying objects in the space around the radar. The radar mathematical model requires local coordinates systems for all objects and the global stationary coordinates system.

The radar station model geometry is based on the Global Coordinates System (GCS) where all objects of the model are described. Figure 2 contains the Cartesian GCS. The plane $x 0 y$ coincides with the ground surface. The radar station is placed at the origin. The vector $0 T$ is directed to the target that has the azimuth $\alpha_{T}$, the elevation $\beta_{T}$ and the angles of deviation of the survey line from coordinate axes $\theta_{x}, \theta_{y}, \theta_{z}$.

The unit vector directed on a target is defined by the direction cosines:

$$
\stackrel{\mathrm{r}}{e_{T}}=\stackrel{\perp}{i} \cdot \cos \theta_{x}+\stackrel{\perp}{j} \cdot \cos \theta_{y}+\stackrel{\perp}{k} \cdot \cos \theta_{z},
$$

where $\stackrel{\perp}{i}, \stackrel{\perp}{j}, \stackrel{\perp}{k}$ are the orts of the coordinates axes $x, y, z$.

The target position is described the Cartesian coordinates $x_{T}, y_{T}, z_{T}$ and its motion is described by the velocity vector projections $v_{T}, v_{T}, v_{T}$. The target azimuth and elevation are found from the simple expressions:

$$
\operatorname{tg} \alpha_{T}=\frac{x_{T}}{y_{T}}, \quad \sin \beta_{T}=\frac{z_{T}}{R_{T}},
$$

where $R_{T}=\sqrt{x_{T}^{2}+y_{T}^{2}+z_{T}^{2}}$ is the target distance.

The antenna array orientation is shown in Figure 3. The flat antenna has its own coordinates system formed by the axes $x^{\prime}, y^{\prime}, z^{\prime}$. Antenna elements are placed on the plane $y^{\prime} 0 z^{\prime}$. The axis $x^{\prime}$ coincides with the normal to the antenna plane with the azimuth $\alpha_{a}$ and the elevation $\beta_{a}$. The target azimuth $\alpha_{T}^{\prime}$ and elevation $\beta_{T}^{\prime}$ in the Antenna Array Coordinates System (AACS) is

$$
\alpha_{T}^{\prime}=\alpha_{T}-\alpha_{a}, \quad \beta_{T}^{\prime}=\beta_{T}-\beta_{a} .
$$

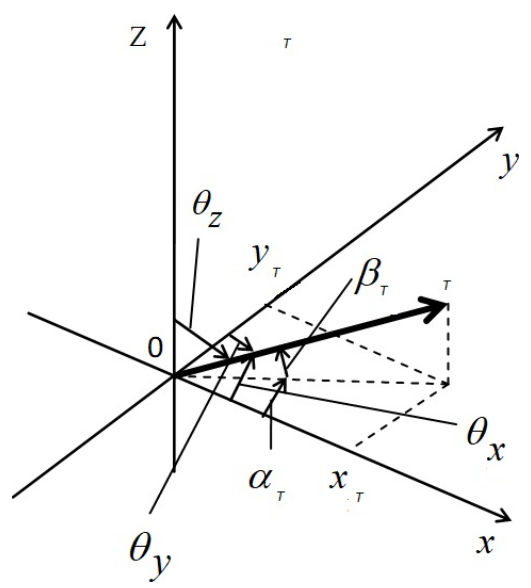

Figure 2: Global coordinates system. 


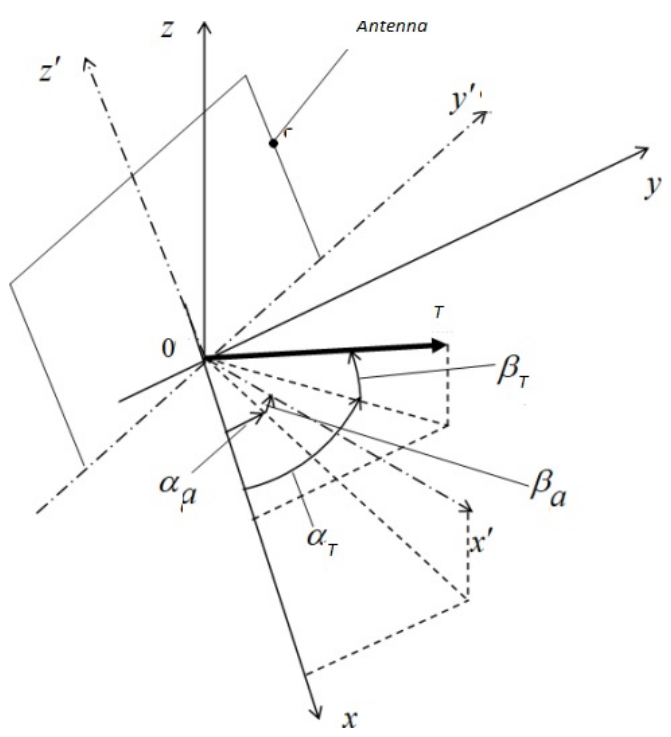

Figure 3: Model global coordinates system.

The values $\alpha_{T}^{\prime}$ and $\beta_{T}^{\prime}$ are used further for calculation of delays on different elements of an antenna array. The delays are necessary for simulation of space-time signal processing.

\section{Target signal simulation with respect to the Doppler effect}

The real radar operation includes some natural processes that require special algorithms of simulation. For example, such process takes place when a received signal is influenced by the Doppler effect. The model gives possibility to set the target motion parameters that help to calculate the radial velocity $V_{r}$. The Doppler frequency is calculated as

$$
\omega_{D}=\omega_{0} \cdot \frac{2 V_{r}}{c}=\frac{4 \pi V_{r}}{\lambda} .
$$

The radial velocity is found from the current target coordinates $x, y, z$ and the velocity projections $V_{x}, V_{y}, V_{z}$ in the GCS:

$$
V_{p}=\frac{x \cdot V_{x}+y \cdot V_{y}+z \cdot V_{z}}{\sqrt{x^{2}+y^{2}+z^{2}}} .
$$

Here is the wideband radio pulse train at the input of one of the antenna elements.

$$
\begin{aligned}
& s_{i}\left(t-(i-1) T_{n}\right)=S\left(t-(i-1) T_{r}\right) . \\
& \quad \cdot \cos \left[\omega_{0}\left(t-(i-1) T_{r}\right)+\omega_{D} t+\psi\left(t-(i-1) T_{r}\right)+\phi_{i}+\gamma\right], \quad i=\overline{1, I},
\end{aligned}
$$

where $I$ is the number of pulses in a pulse train, $T_{r}$ is the repetition period. All pulses in (6) are supposed to have the same amplitude and angle modulation, carrier frequency $\omega_{0}$ and Doppler frequency $\omega_{D}$, phase incursion $\gamma$. The independent random initial phases $\phi_{i}$ are caused by a 
clock generator. With respect to coherent heterodyne properties the initial phase is compensated at the intermediate frequency:

$$
\begin{aligned}
s_{I F i}(t) & =S\left(t-(i-1) T_{r}\right) . \\
& \cdot \cos \left[\omega_{I F}\left(t-(i-1) T_{r}\right)+\omega_{D} t+\psi\left(t-(i-1) T_{r}\right)+\gamma\right], \quad i=\overline{1, I} .
\end{aligned}
$$

As the SRS simulation is based on complex envelopes the signals in (7) are represented in the following form:

$$
\begin{aligned}
s_{I F i}(t) & =\operatorname{Re}\left\{S\left(t-(i-1) T_{r}\right) e^{j\left[\omega_{I F}\left(t-(i-1) T_{r}\right)+\omega_{D} t+\psi\left(t-(i-1) T_{r}\right)+\gamma\right]}\right\}= \\
& =\operatorname{Re}\left\{S^{\&}\left(t-(i-1) T_{r}\right) e^{j \omega_{D} t} e^{j\left(\omega_{I F}\left(t-(i-1) T_{r}\right)+\gamma\right)}\right\}, \quad i=\overline{1, I} .
\end{aligned}
$$

where $S^{\&}\left(t-(i-1) T_{r}\right)=S\left(t-(i-1) T_{r}\right) e^{i \psi\left(t-(i-1) T_{r}\right)}$ is the complex envelope of the $i$-th pulse without the Doppler effect. The multiplier $e^{j \omega_{D} t}$ describing the Doppler effect is a slow time function. Hence it can be replaced by the complex coefficients $e^{j \omega_{D}(i-1) T_{r}}$ :

$$
S_{I F}^{\&}\left(t-(i-1) T_{r}\right)=S^{\&}\left(t-(i-1) T_{r}\right) e^{j \omega_{D}(i-1) T_{r}} .
$$

The FFT of (9) gives possibility to find the spectrum of the complex envelope of a received signal with respect to the Doppler effect as the complex envelope spectrum without Doppler effect multiplied by the complex coefficient

$$
k_{i}=e^{j \omega_{D}(i-1) T_{r}} .
$$

When the Doppler effect is simulated, the Doppler frequency can be multiple of the repetition frequency of the sounding signal when a passive interference is suppressed together with a useful signal. This leads to the noticeable decrease of the target detection probability.

\section{Simulation of DPAA direction characteris}

DPAA direction characteristics for the received signal are formed adaptively to achieve maximum suppression of active interferences. The research described in this paper is based on the methods of the theory of multidimensional signals and their space-time processing. There is no direct description of antenna characteristics in such approach. The space-time approach is necessary because wideband signals are used. A complex envelop of a wideband signal is not the same on different antenna elements.

Simulation of a sounding signal can be implemented with common characteristics of antennas. Further there are main expressions for simulation of the primary signal in the point of a target or of reflectors creating a passive interference.

The directional diagram of a transmitting antenna is

$$
G\left(\alpha, \alpha_{0}, \beta, \beta_{0}\right)=G_{0}\left(\alpha_{0}, \beta_{0}\right) \cdot g\left(\frac{\alpha-\alpha_{0}}{\alpha_{0,5}\left(\alpha_{0}\right)}, \frac{\beta-\beta_{0}}{\beta_{0,5}\left(\beta_{0}\right)}\right)
$$


where $\alpha_{0}, \beta_{0}$ are the azimuth and elevation describing antenna diagram beam deviation because of electronic scanning relative to the normal to the antenna array surface, $\alpha_{0,5}\left(\alpha_{0}\right), \beta 0,5\left(\beta_{0}\right)$ are the beam widths on the azimuth and elevation:

$$
\alpha_{0,5}\left(\alpha_{0}\right)=\frac{51 \lambda}{N_{\alpha} d \cos \alpha_{0}}, \quad \beta_{0,5}\left(\beta_{0}\right)=\frac{51 \lambda}{N_{\beta} d \cos \beta_{0}},
$$

where $N_{\alpha}, N_{\beta}$ are the numbers of antenna elements rows and colons in a flat antenna array, $\lambda$ is the carrier wavelength, $d$ is the distance between antenna elements. If $d=\lambda / 2$, then (12) takes the form:

$$
\alpha_{0,5}\left(\alpha_{0}\right)=\frac{102}{N_{\alpha} \cos \alpha_{0}}, \quad \beta_{0,5}\left(\beta_{0}\right)=\frac{102}{N_{\beta} \cos \beta_{0}},
$$

The results of calculations by (12) and (13) are expressed in degrees. If $\alpha_{0}=\beta_{0}=0$, then the beam width is minimum. If beam deviation is nonzero the directional diagram is expanded.

The parameter $G_{0}\left(\alpha_{0}, \beta_{0}\right)$ in (11) describes the antenna gain. It is evident that if the angles $\alpha_{0}, \beta_{0}$ grow the gain falls. It depends on the effective antenna surface $S_{\text {eff }}$

$$
G_{0}\left(\alpha_{0}, \beta_{0}\right)=4 \pi \frac{S_{e f f}}{\lambda^{2}},
$$

which is found from the expression:

$$
S_{e f f}=N_{\alpha} d \cos \alpha_{0} N_{\beta} d \cos \beta_{0}=N_{\alpha} N_{\beta} d^{2} \cos \alpha_{0} \cos \beta_{0} .
$$

With respect to (15) and $d=\lambda / 2$, the expression (14) takes the form:

$$
G_{0}\left(\alpha_{0}, \beta_{0}\right)=\pi N_{\alpha} N_{\beta} \cos \alpha_{0} \cos \beta_{0} .
$$

The multiplier $g\left(\frac{\alpha-\alpha_{0}}{\alpha_{0,5}\left(\alpha_{0}\right)}, \frac{\beta-\beta_{0}}{\beta_{0,5}\left(\beta_{0}\right)}\right)$ of (11) is a normalized directional diagram with $g(0,0)=1$. It is approximated by the function $\sin \gamma / \gamma$ :

$$
g\left(\frac{\alpha-\alpha_{0}}{\alpha_{0,5}\left(\alpha_{0}\right)}, \frac{\beta-\beta_{0}}{\beta_{0,5}\left(\beta_{0}\right)}\right)=\left|\frac{\sin \left(3.79 \frac{\alpha-\alpha_{0}}{\alpha_{0,5}\left(\alpha_{0}\right)}\right)}{3.79 \frac{\alpha-\alpha_{0}}{\alpha_{0,5}\left(\alpha_{0}\right)}} \cdot \frac{\sin \left(3.79 \frac{\beta-\beta_{0}}{\beta_{0,5}\left(\beta_{0}\right)}\right)}{3.79 \frac{\beta-\beta_{0}}{\beta_{0,5}\left(\beta_{0}\right)}}\right| .
$$

The normalized directional diagram is featured by the properties:

$$
g(0.5 ; 0)=g(0 ; 0.5)=0.5 .
$$

Its level decreases on each angle coordinate until the half level.

\section{Testing of the model of a radar station with DPAA}

The program for simulation of an SRS has been approved by experiments. The main attention is focused on the influence of active interferences on the target detection probability. As a 
Table 1

Target detection probability for $10 \mathrm{Al}$.

\begin{tabular}{c|ccccc}
\hline$N_{A I}, \mathrm{~kW} / \mathrm{MHz}$ & 10 & 10 & 10 & 1 & 1 \\
$R_{T}, \mathrm{~km}$ & 300 & 250 & 200 & 300 & 250 \\
$q_{D}, \mathrm{~dB}$ & -91.4 & -88 & -84.4 & -81 & -78.3 \\
$q_{F}, \mathrm{~dB}$ & -8.4 & -1.9 & 1.8 & 1.6 & 4.6 \\
$P_{D}$ & 0.01 & 0.1 & 1 & 0.8 & 1 \\
\hline
\end{tabular}

Table 2

Al angle coordinates.

\begin{tabular}{c|cccccccccc}
\hline$\alpha_{A I},{ }^{\circ}$ & 10 & 15 & 20 & 25 & 30 & 40 & 50 & 60 & 70 & 80 \\
$\beta_{A I},{ }^{\circ}$ & 7 & 9 & 5 & 7 & 10 & 5 & 5 & 6 & 8 & 5 \\
\hline
\end{tabular}

target signal and active interferences income from different directions their spectrums are also different. Hence, it is possible to suppress interferences. Further there are the results of the experiment with a simulated target and 10 active interference sources. This experiment includes simulation of space-time filtration and target detection for the following parameters: ESS $\sigma_{T}=1 \mathrm{~m}^{2}$, distance $R_{T}=300 \mathrm{~km}$, angle coordinates $\left(\alpha_{T}, \beta_{T}\right)=(0,0)^{\circ}$, false alarm probability $F=10^{-6}$, transmitter power $P_{T R}=80 \mathrm{~kW}$, signal type is the radio pulse with the linear frequency modulation with pulse width $\tau_{P}=10 \mu \mathrm{s}$, frequency deviation $\Delta f=$ $100 \mathrm{MHz}$, number of pulses $N_{p}=5$, noise PSD $N_{N}=10^{-20} \mathrm{~W} / \mathrm{Hz}$, Active Interference (AI) PSD $N_{A I}=\{0.1,1,10\} \mathrm{kW} / \mathrm{MHz}$, AI bandwidth $\Delta F_{A I}=100 \mathrm{MHz}$, distance until AI sources $R_{A I}=350 \mathrm{~km}$, the member of DPAA elements $70 \times 70=4900$, sample frequency $f_{s}=200 \mathrm{MHz}$, the statistical experiment volume is $N_{E}=100$.

The experiment results include estimations of the detection probability $P_{D}$ and Signal to Noise Plus Interference Ratio (SNPIR) values at the DPAA input $q_{A}$ and at the output of the Space-Time Matching Filter (STMF) $q_{F}$.

Table 1 contains the experiment results for $10 \mathrm{AI}$ sources. The angle coordinates of the AI sources are shown in Table 2. The SNPIR improvement at the STMF output is about $83 \mathrm{~dB}$. If $R_{T}=300 \mathrm{~km}$ and $N_{A I}=10 \mathrm{~kW} / \mathrm{MHz}$ the detection probability is about zero. The stable detection of a target takes place if $R_{T}=200 \mathrm{~km}$. If $N_{A I}=10 \mathrm{~kW} / \mathrm{MHz}$ and $R_{T}=250 \mathrm{~km}$, the detection probability is equal to one. If $R_{T}=250 \mathrm{~km}$, the detection probability is 0.8 .

\section{Conclusion}

The proposed model gives possibility to analyze the algorithms for radar signal processing in SRS with DPAA. The model speed primary depends on the computer RAM volume, sounding signal width and interferences number. The satisfactory calculation speed is achieved by simulation of only active intervals of transmitted and received radar signals at zero frequency. The model capabilities are approved for many AI sources. 


\section{References}

[1] Mahafza B.R., Elshebeni A.Z. Matlab simulations for radar systems design. Chapman \& Hall/CRC CRC Press LLC, 2004. 686 p.

[2] Curry G.R. Radar systems performance modeling. Artech House, Inc., 2005. $411 \mathrm{p}$

[3] Berry P.E., Currie G., Yau D. Generic phased array radar model for detailed radar performance assessment // 19th International Congress on Modelling and Simulation. Perth, Australia, 12-16 December 2011. P. 440-446.

[4] Bystrov R.P., Korniyenko V.N., Kuzmichev V.E., Pozhidayev V.N., Sokolov S.A., Cherepnin V.A. Methods of simulation of terahertz radar systems // Journal of Radio Electronics. 2015. No. 7. Available at: http://jre.cplire.ru/jre/jul15/9/text.html. (In Russ.)

[5] Kalinin T.V., Bartsevich A.V., Petrov S.A., Khrestinin D.V. Program complex for simulation of recognition radar system / Software \& Systems. 2017. No. 4. P. 733-738. (In Russ.)

[6] Diewald A.R., Culotta-López C. Concepts for radar target simulation // Loughborough Antennas \& Propagation Conference (LAPC 2017). Loughborough, 2017. P. 1-5.

[7] Hao L.L., Yanzhong C.H., Yanchun L., Hui L., Ming L. Studies on phased-array radar jamming and simulation system // Applied Mechanics and Materials. Vol. 385-386. P. 1476-1479.

[8] Haws L.A. Baseband digital signal processing of radar system // PLTOFF, School of Engineering \& Information Technology. ZEIT4501, 2015. P. 1-13.

[9] $\mathrm{Wu} \mathrm{H}$. Simulation of radar signal processing based on Matlab // Information Science and Management Engineering IV (ISME 2016). 2016. P. 299-304.

[10] Shirman Y.D. Leshchenko S.P., Orlenko V.M. Advantages and problems of wideband radar // 2003 Proceedings of the International Conference on Radar (IEEE Cat. No. 03EX695). Adelaide, SA, Australia, 2003. P. 15-21.

[11] Afzal M.U., Qureshi A.A., Tarar M.A., Tauqeer T. Analysis, design, and simulation of phased array radar front-end // 2011 7th International Conference on Emerging Technologies. Islamabad, Pakistan, 2011. P. 1-6.

[12] Zhao T., Dong C., Ren H., Yin H. The radar echo simulation of moving targets based on HRRP // 2013 IEEE International Conference on Green Computing and Communications and IEEE Internet of Things and IEEE Cyber, Physical and Social Computing. Beijing, China, 2013. P. 1580-1583.

[13] Ergezer H., Furkan Keskin M., Gunay O. Real-time radar, target, and environment simulator / Obaidat M., Ören T., Kacprzyk J., Filipe J. (eds) // Simulation and Modeling Methodologies, Technologies and Applications. Advances in Intelligent Systems and Computing. Springer, Cham, 2015. Vol. 402. DOI:10.1007/978-3-319-26470-7_11.

[14] Nativ Y. RADAR simulation. MATLAB central file exchange. Available at: https://www. mathworks.com/matlabcentral/fileexchange/13485-radar-simulation (accessed June 01, 2021).

[15] Senanayaka D. Simple radar system simulation. MATLAB central file exchange. Available at: https://www.mathworks.com/matlabcentral/fileexchange/25631-simple-radar_system_ simulation (accessed June 01, 2021).

[16] Nogra B. Radar basic simulation. MATLAB central file exchange. Available at: https:// www.mathworks.com/matlabcentral/fileexchange/75323-radar-basic-simulation (accessed 
June, 2021)

[17] Lozovskiy I.F., Murasev A.A., Spektor A.A. Radar detection of concentrated objects using broadband radiation // CEUR Workshop Proceedings. 2020. Vol. 2534. P. 132-136.

[18] Morozov Yu.V., Rajfeld M.A., Spektor A.A. Non-parametric target detection in a passive seismic locator based on spectral data // Journal of Theoretical and Applied Information Technology. 2018. Vol. 96. No. 11. P. 3567-3577. 